\title{
Données sur les concentrations en plutonium dans l'écosystème aquatique rhodanien en aval de l'usine de Marcoule
}

\author{
A. LAMBRECHTS*, F. LEVY**, L. FOULQUIER*
}

RÉSUMÉ Le site de Marcoule comprend une usine qui retraite le combustible irradié des réacteurs graphite-gaz et en extrait le plutonium. Une usine de fabrication du combustible MOX est en cours de construction. Ces activités sont génératrices d'effluents liquides rejetés au Rhône après traitement. Pour les émetteurs $\alpha$, la réglementation en vigueur limite les concentrations de ces effluents à 150 GBq/an. Les rejets en plutonium et transplutoniens sont faibles (41 GBq en 1989).

Des mesures de plutonium ont été effectuées en 1989 dans certains compartiments de l'écosystème aquatique rhodanien en aval de l'usine. Au niveau du barrage de Vallabrègues (15 km au sud de Marcoule) on trouve les concentrations en ${ }^{238} \mathrm{Pu}$ et ${ }^{239}+240 \mathrm{Pu}$ respectivement égales à 2,1 et $7,8 \mathrm{~Bq} \cdot \mathrm{kg}^{-1}$ séc dans les sédiments, 1,1 et $3,8 \mathrm{~Bq} \cdot \mathrm{kg}^{-1}$ sec dans les végétaux aquatiques immergés et égales ou inférieures aux limites de détection dans l'eau et les poissons. Dans les sédiments et les végétaux, le rapport ${ }^{238} \mathrm{Pu} /{ }^{239}+240 \mathrm{Pu}$ est d'environ 0,3 . Les concentrations en plutonium des compartiments du milieu diminuent rapidement lorsqu'on s'éloigne de l'usine et sont inférieures aux limites de détection en Carmargue.

SUMMARY The Marcoule site includes a plant reprocessing spent fuel from gas-graphite reactors and extracting plutonium. A plant for the processing of MOX fuel is being built. These plants are at the origin of the release into the Rhone river of liquid effluents once they have been treated. The present regulations limit concentration of alpha-emitters in the releases to $150 \mathrm{~Bq} / \mathrm{y}$.

Plutonium has been measured in 1989 in some compartiments of the Rhone basin aquatic ecosystem downstream. At the level of the Vallabregues dam (15 km south of Marcoule), ${ }^{238} \mathrm{Pu}$ and ${ }^{239}+240 \mathrm{Pu}$ concentrations are respectively 2.1 and $7.8 \mathrm{~Bq} . \mathrm{kg}^{-1}$ dry in sediments, 1.1 and $3.8 \mathrm{~Bq} \cdot \mathrm{kg}^{-1}$ dry in immersed aquatic plants and equal or below detection limits in water and fish. In sediments and plants, ${ }^{238} \mathrm{Pu} / 239+240 \mathrm{Pu}$ ratio is about 0.3 . The farther from the installation, the more quickly plutonium concentrations in the environmental compartments decrease, and they are below detection limits in the Camargue.

* Commissariat à l'énergie atomique, Institut de protection et de sureté nucléaire, DPEI/SERE, Bt 153, Centre de Cadarache, BP n 1, 13108 St-Paul-lez-Durance.

* COGEMA, Service de protection contre les rayonnements, Centre de Marcoule, BP $N^{\circ}$ 170,30200 Bagnols-sur Cèze. 


\section{INTRODUCTION}

Sur le site de Marcoule sont implantées plusieurs installations nucléaires dont des réacteurs (Célestin, Phénix) et une usine qui effectue le retraitement du combustible irradié de la filière des réacteurs à uranium naturel-graphite-gaz. Une usine de fabrication de combustibles MOX ${ }^{1}$ est en cours de construction (usine MELOX). Elle devrait être opérationnelle à partir de 1994.

Ces activités sont génératrices d'effluents liquides qui sont rejetés dans le Rhône après traitement, dans le respect de la législation en vigueur (arrêté du 21 mai 1981). La Compagnie générale de matières nucléaires (COGEMA) a décidé d'effectuer, en complément de la surveillance de l'environnement exercée par son Service de protection contre les rayonnements (SPR), un suivi radioécologique du Rhône entre l'amont de Marcoule et son embouchure en Méditerranée. Cette étude, réalisée en collaboration entre la COGEMA et l'Institut de protection et de sûreté nucléaire (IPSN) s'est attachée, entre autres objectifs, à évaluer l'impact des effluents émetteurs $\alpha$ sur les compartiments de l'écosystème aquatique rhodanien.

\section{CONDITIONS DE REJETS AU RHÔNE D'ÉFFLUENTS ÉMETTEURS $\alpha$ PAR L'USINE DE MARCOULE}

L'arrêté d'autorisation de rejet d'effluents liquides (Journal officiel du 21 mai 1981, N.C. 4957) impose de ne pas dépasser une activité annuelle de $150 \mathrm{GBq}$ de radionucléides émetteurs $\alpha$. L'activité volumique ajoutée, calculée après dilution totale dans le Rhône doit être au maximum, en valeur moyenne quotidienne, de $40 \mathrm{mBq} .1^{-1}$. Le débit du Rhône doit être compris entre 400 et $4000 \mathrm{~m}^{3} \cdot \mathrm{s}^{-1}$ sinon une demande d'accord préalable au Service central de protection contre les rayonnements ionisants (SCPRI) est nécessaire. L'activité des rejets effectués dans un mois doit être inférieure au 1/6 des limites annuelles.

Selon leurs caractéristiques physico-chimiques, les effluents sont traités par évaporation ou coprécipitation et décantation. Après passage sur filtre de $25 \mu \mathrm{m}$, les eaux résiduelles sont rejetées au Rhône. Les radionucléides, à l'exception du ruthénium, sont rejetés sous forme de nitrates.

Les rejets se font au point kilométrique ${ }^{2}$ (PK) 210,5 par une clarinette comportant 12 buses dont la forme et l'orientation sont calculées pour que la dilution soit complète en $600 \mathrm{~m}$. Une station de contrôle se trouve au PK 222. En 1989, les rejets en plutonium et transplutoniens ont été de $41 \mathrm{GBq}$.

1. Mélange d'oxydes de plutonium et d'uranium.

2. Les points kilométriques sont calculés à partir de la confluence du Rhône et de la Saône où se trouve le service de la navigation (PK 0). L'usine de Marcoule est située au PK 208,5. 


\section{MÉTHODE DE PRÉLÈVEMENT ET DE PRÉPARATION DES ÉCHANTILLONS}

Cent litres d'eau sont prélevés et concentrés par évaporation, 8 I de sédiment sont prélevés au cône de Berthois. Une fraction est séchée à $110^{\circ} \mathrm{C}$ pour les mesures de radioactivité.

Les végétaux aquatiques sont prélevés à partir de la berge à l'aide de gaffes ou de grappins. Ils sont rincés dans l'eau du fleuve et mis dans des sacs plastiques pour être ramenés au laboratoire. Ils sont soigneusement lavés à l'eau du robinet, pesés frais et séchés à $110^{\circ} \mathrm{C}$ jusqu'à obtention d'un poids constant. lls sont ensuite incinérés dans un four dont la montée en température jusqu'à $560^{\circ} \mathrm{C}$ est programmée sur un cycle de $24 \mathrm{~h}$.

Les poissons sont prélevés par pêche électrique à partir d'un bateau spécialement équipé. Ils sont séparés par espèces, mesurés et pesés frais. Leur traitement est identique à celui des végétaux aquatiques.

Les analyses d'émetteurs $\alpha$ ont été effectuées par le laboratoire du Service mixte de sécurité radiologique (BP $n^{\circ} 16,91311$ Monthléry) qui effectue les dosages de plutonium et d'américium par radiochimie et spectrométrie alpha à chambre à grille. Les limites de détection sont de $0,2 \mathrm{~Bq} \cdot \mathrm{kg}^{-1}$ de matière sèche ou calcinée et de $5 \cdot 10^{-3} \mathrm{~Bq} \cdot \mathrm{m}^{-3}$ d'eau.

Les résultats des mesures sont exprimés en Bq..$^{-1}$ pour l'eau, en Bq. $\mathrm{kg}^{-1}$ sec pour les sédiments et les végétaux, en Bq. $\mathrm{kg}^{-1}$ frais pour les poissons. Le tableau I permet d'effectuer les calculs pour comparer les résultats avec ceux d'autres auteurs.

TABLEAU I

Rapports poids frais/poids sec des végétaux aquatiques et des poissons des rivières françaises

\begin{tabular}{|cc|}
\hline Phanérogames immergées & $13,93 \pm 0,88(286)$ \\
Mousses aquatiques & $7,56 \pm 0,63(172)$ \\
Poissons & $3,85 \pm 0,10(2307)$ \\
\hline
\end{tabular}

(Dans ce tableau, comme dans les suivants, les valeurs moyennes présentées sont affectées de leur intervalle de confiance, correspondant à une probabilité de $95 \%$, calculé par la formule $m=\bar{x} \pm t . \frac{s}{\sqrt{n}}$ où test la variable de Student, s est l'écart-type population et $n$ est le nombre de valeurs significatives indiqué entre parenthèses). 
Les données relatives aux prélèvements, au conditionnement et aux résultats de mesures sont gérées sur un ordinateur IBM 370 du réseau CEA (CEANET).

\section{RADIOACTIVITÉ $\alpha$ DES COMPARTIMENTS DU FLEUVE}

\subsection{Radioactivité de l'eau}

La radioactivité $\alpha$ totale de l'eau a été mesurée par le SPR de Marcoule de 1961 à 1990 au niveau de l'usine et à Roquemaure. Elle oscille entre 20 et $40 \mathrm{mBq} .1^{-1}$ due principalement à l'uranium naturel. Des échantillons ont été prélevés à l'écluse de Vallabrègues et dans le canal d'amenée à Fourques en août 1989 et en mai 1990 par le Laboratoire de radioécologie des eaux continentales de Cadarache.

Les valeurs obtenues en spectrométrie alpha sont respectivement de 0,02 et $0,06 \mathrm{mBq} \cdot \mathrm{I}^{-1}$ pour ${ }^{238} \mathrm{Pu}$ et $239+240 \mathrm{Pu}$, tant à Vallabrègues qu'à Fourques, avec le rapport de 0,3 caractéristique de l'usine (tableau II).

TABLEAU II

Radioactivité $\alpha$ du plutonium de l'eau prélevée en aval de Marcoule en août 1989 (mBq..$^{-1}$ )

\begin{tabular}{|ccc|}
\hline N & Vallabregues & courques \\
\hline $328 \mathrm{Pu}$ & $0,018 \pm 0,010$ & $<0,023$ \\
$239+240 \mathrm{Pu}$ & $0,058 \pm 0,014$ & $0,063 \pm 0,012$ \\
$328 \mathrm{Pu} / 239+240 \mathrm{Pu}$ & 0,31 & $<0,35$ \\
\hline
\end{tabular}

\subsection{Radioactivité des sédiments}

De Marcoule à Vallabrègues on trouve un sédiment fin ayant plus de $70 \%$ de particules inférieures à $50 \mu \mathrm{m}$. Dans le canal du Bas-Rhône, ces particules fines ne représentent plus que $45 \%$ du sédiment. Dans la partie inférieure du Rhône, leur proportion est de l'ordre de $35 \%$. Ils sont riches en minéraux gonflants (smectites et illites).

Des mesures de $239+240 \mathrm{Pu}$ effectuées en 1986 donnaient des concentrations de $0,005 \mathrm{~Bq} \cdot \mathrm{kg}^{-1} \mathrm{sec}$ en amont de l'usine et 0,7 en aval [5]. 
Les résultats obtenus en 1989 sont consignés dans le tableau III.

TABLEAU III

Radioactivité $\alpha$ du plutonium dans des sédiments prélevés en septembre 1989, en amont et en aval du site de Marcoule

(en Bq. $\mathrm{kg}^{-1}$ sec)

\begin{tabular}{|c|c|c|c|}
\hline lle vieille & $\leq 0,13$ & $\leq 0,11$ & - \\
\hline Marcoule amont & $\leq 0,15$ & $\leq 0,12$ & - \\
\hline$\cdots \cdots$ & $=\ldots \ldots$ & 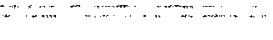 & \\
\hline Marcoule aval & $18,20 \pm 1,40$ & $50,60 \pm 3,60$ & 0,36 \\
\hline Caderousse rive droite & $0,35 \pm 0,10$ & $0,95 \pm 0,14$ & 0,36 \\
\hline Ecluse de Vallabrègues & $2,13 \pm 0,23$ & $7,83 \pm 0,64$ & 0,27 \\
\hline Fourques, chambre & $1,97 \pm 0,20$ & $6,96 \pm 0,54$ & 0,28 \\
\hline Pont de Belleval & $1,24 \pm 0,17$ & $4,27 \pm 0,38$ & 0,29 \\
\hline Mas Thibert & $0,43 \pm 0,12$ & $1,11 \pm 0,16$ & 0,39 \\
\hline La Palissade & $0,16 \pm 0,10$ & $0,43 \pm 0,10$ & 0,37 \\
\hline Pont de Sylvéréal & $0,17 \pm 0,10$ & $0,81 \pm 0,12$ & 0,21 \\
\hline Pont de Saint-Gilles & $0,88 \pm 0,14$ & $2,96 \pm 0,29$ & 0,29 \\
\hline Le Sauvage & $0,69 \pm 0,12$ & $2,14 \pm 0,22$ & 0,32 \\
\hline Canal de Fumemorte & $\leq 0,15$ & $\leq 0,15$ & - \\
\hline Vaccarès & $\leq 0,15$. & $0,14 \pm 0,14$ & - \\
\hline Canal du Rousty & $\leq 0,19$ & $0,20 \pm 0,08$ & - \\
\hline
\end{tabular}

Les concentrations en ${ }^{238} \mathrm{Pu}$ et $239+240 \mathrm{Pu}$ sont maximales à l'aval immédiat de l'usine pour se maintenir aux environs de 2 et $7 \mathrm{~Bq} / \mathrm{kg} \mathrm{sec}$ entre Vallabrègues et Fourques. Dans la partie la plus aval du Rhône la concentration en plutonium des sédiments se réduit encore et devient inférieure aux niveaux de détection dans les canaux de la Camargue et l'étang du Vaccarès. Dans toute la zone du Rhône située en aval de l'usine de Marcoule le rapport ${ }^{238} \mathrm{Pu} / 239+240 \mathrm{Pu}$ est compris entre 0,2 et 0,4 (moyenne 0,31), ce qui est bien caractéristique de l'usine de retraitement. Ce rapport est confirmé par J.M. Martin \& A.J. Thomas [7] qui trouvent une valeur de 0,24 en aval de Marcoule. Pour ces auteurs, ce rapport est de 0,03 à 0,05 dans les sédiments prélevés en amont de toute installation nucléaire. 


\subsection{Radioactivité des végétaux aquatiques}

De 1980 à 1986 des mesures du plutonium effectuées ${ }^{1}$ sur des végétaux prélevés en aval de Marcoule avaient donné des concentrations moyennes de $0,9 \pm 0,5 \mathrm{~Bq} \cdot \mathrm{kg}^{-1} \mathrm{sec}$ en ${ }^{238} \mathrm{Pu}$ et de $2,4 \pm 1,4 \mathrm{~Bq} \cdot \mathrm{kg}^{-1} \mathrm{sec}$ en $239+240 \mathrm{Pu}$.

Une campagne de prélèvements de végétaux aquatiques s'est déroulée du 28 au 31 août 1989. Les plantes prélevées sont de bryophytes (mousses) et des phanérogames (plantes à fleurs) immergées. Les valeurs en plutonium figurent dans le tableau IV.

TABLEAU IV

Radioactivité $\alpha$ du plutonium dans les végétaux prélevés en août 1989 en amont et en aval du site de Marcoule

(en Bq. $\mathrm{kg}^{-1} \mathrm{sec}$ )

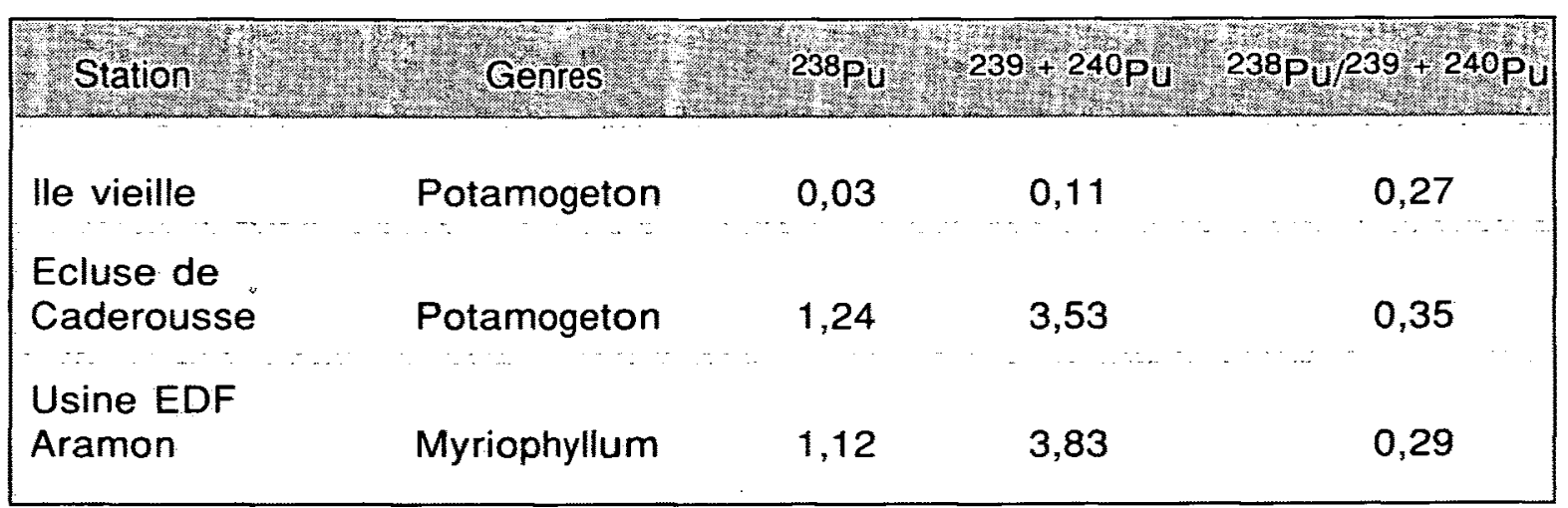

Le rapport des ${ }^{238} \mathrm{Pu} / 239+{ }^{240} \mathrm{Pu}$ est de 0,33 et correspond à celui que l'on mesurait dans les sédiments. Le SPR de Marcoule trouve, dans les végétaux prélevés en septembre 1989, des concentrations en ${ }^{239} \mathrm{Pu}$ inférieures à $10 \mathrm{~Bq} \cdot \mathrm{kg}^{-1} \mathrm{sec}$ (tableau V).

\subsection{Radioactivité des poissons}

Des mesures de plutonium effectuées sur des poissons prélevés entre 1980 et 1986 en aval de Marcoule donnaient des valeurs moyennes en ${ }^{238} \mathrm{Pu}$ et $239+240 \mathrm{Pu}$ respectivement inférieures à 0,03 et $0,05 \mathrm{~Bq} \cdot \mathrm{kg}^{-1}$ frais. Des pêches électriques ont été effectuées entre février et septembre 1989 en amont et en aval de l'usine de Marcoule. Les poissons présentent des concentrations en ${ }^{238} \mathrm{Pu}$ et $239+240 \mathrm{Pu}$ inférieures à la limite de détection (tableau VI).

1. Mesures effectuées par le Laboratoire d'analyses radiotoxicologiques et de biochimie du Département de protection sanitaire du CEA, Fontenay-aux-Roses. 
TABLEAU $V$

Concentration en plutonium des végétaux prélevés en amont et en aval de Marcoule par le SPR en septembre 1989 (en $\mathrm{Bq} \cdot \mathrm{kg}^{-1} \mathrm{sec}$ )

\begin{tabular}{|ccc|}
\hline Station (PK) & Espéce & $239+240 \mathrm{Pu}$ \\
\hline Amont usine (202) & Myriophylle & $<5,2$ \\
Amont usine (202) & Potamot & $<4,8$ \\
Amont usine (202) & Mousse & $<15,2$ \\
Amont usine (212) & Myriophylle & $<6,3$ \\
Amont usine (212) & Potamot & 9,6 \\
Caderousse (218) & Myriophylle & $<5,6$ \\
Villeneuve (240) & Myriophylle & 5,6 \\
Petit Rhône (280) & Myriophylle & $<4,4$ \\
Petit Rhône (316) & Myriophylle & $<7,8$ \\
Petit Rhône (331) & Myriophylle & $<9,3$ \\
\hline
\end{tabular}

TABLEAU VI

Concentration en plutonium des poissons prélevés en amont et en aval de Marcoule (en Bq. $\mathrm{kg}^{-1}$ sec)

\begin{tabular}{|lllll|}
\hline Station & Genres & Date & $238 \mathrm{Pu}$ & $239+240 \mathrm{Pu}$ \\
\hline Marcoule amont & Chevaine & $89 / 09 / 14$ & $\leq 0,1$ & $\leq 0,1$ \\
Marcoule aval & Anguille & $89 / 09 / 14$ & $\leq 0,1$ & $\leq 0,1$ \\
\hline
\end{tabular}

\section{CONCLUSION}

En aval de l'usine de Marcoule, les concentrations moyennes des compartiments de l'écosystème aquatique en plutonium sont faibles comme le montrent les valeurs mesurées, en 1989, au niveau du barrage de Vallabrègues, $50 \mathrm{~km}$ en aval de l'usine (tableau VII). A titre comparatif, l'Organisation mondiale de la santé (OMS) fixe une limite de concentration en ${ }^{241} \mathrm{Am}-{ }^{239} \mathrm{Pu}$ dans les aliments soumis au commerce interna- 
tional égale à $10 \mathrm{~Bq} \cdot \mathrm{kg}^{-1}$ pour les aliments de consommation générale et de $1 \mathrm{~Bq} . \mathrm{I}^{-1}$ pour le lait donné aux nourrissons [9].

TABLEAU VII

Concentration en plutonium

des compartiments de l'écosystème aquatique rhodanien

au niveau du barrage de Vallabrègues, $50 \mathrm{~km}$ en aval de l'usine

\begin{tabular}{|lcc|}
\hline & $238 \mathrm{pu}$ & $239+240 \mathrm{Pu}$ \\
\hline Eau $\left(\mathrm{mBq} \cdot \mathrm{I}^{-1}\right)$ & $<0,02$ & $<0,02$ \\
\hline Sédiments $\left(\mathrm{mBq} \cdot \mathrm{kg}^{-1} \mathrm{sec}\right)$ & 2,1 & 7,8 \\
\hline Végétaux $\left(\mathrm{mBq} \cdot \mathrm{kg}^{-1} \mathrm{sec}\right)$ & 1,1 & 3,8 \\
\hline Poissons (mBq.kg $\left.{ }^{-1} \mathrm{sec}\right)$ & $<0,1$ & $<0,1$ \\
\hline
\end{tabular}

Les concentrations en plutonium des compartiments diminuent en fonction de la distance de la station de prélèvement au point de rejet. Au niveau de la Camargue, elles sont inférieures aux limites de détection.

Bien qu'elle soit implantée depuis 30 ans, l'usine de Marcoule a faiblement marqué en émetteurs $\alpha$ les compartiments du Rhône.

\section{RÉFÉRENCES}

[1] BOVARD P., GRAUBY A., FOULQUIER L., PICAT Ph. - Etude radioécologique du bassin rhodanien : stratégie et bilan. In : Environmental behaviour of radionuclides released in the nuclear industry, Aix-en-Provence, 14-18 May 1973. Vienne : AIEA, 1973, 507-523.

[2] DESCAMPS B., BAUDIN-JAULENT Y. - Transfer or radium 226 to fish in the aquatic environment of a french mining complex, assessment of radiation dose to man. In: The cycling of long-lived radionuclides in the biosphere: observations and models, Madrid, 15-19 septembre 1986. Luxembourg : CCE, 1987, vol. 2, $17 \mathrm{p}$.

[3] ESTOURNEL R., GALISSIAN F. MARICHAL M. - Les rejets de Marcoule dans le Rhône et leur contrôle. Rev. Inst. Pasteur, Lyon,. 1967-1968, 1 (5), 831-834.

[4] FOULQUIER L., PALLYM. - Données radioécologiques sur les sédiments du BasRhône. Sci. Eau, 1984, 3 (3), 259-277.

[5] FOULQUIER L., LAMBRECHTS A., PALLY M. - Impact radioécologique d'une usine de retraitement de combustibles nucléaires sur un fleuve : le Rhône. In : 
Nuclear fuel reprocessing and waste management, Paris, Aug. 23-27, 1987. Paris : Société française d'énergie nucléaire, 1988, vol. 3, 1063-1071.

[6] LAMBRECHTS A., FOULQUIER L. - Radioecology of the Rhône basin : data on the fish of the Rhône (1974-1984). J. Environ. Radioact., 1987, 5 (2) : 105-121.

[7] MARTIN J.M., THOMAS A.J. - Contamination radioactive de l'environnement par l'industrie nucléaire. In : Actes du Colloque "Nucléaire-santé-sécurité", Montauban, 21-23 janvier 1988, Montauban : Conseil général du Tarn et Garonne, 1989, 347389.

[8] MARTIN J.M., THOMAS A.J. - Origins, concentrations and distributions of artificial radionuclides discharged by the Rhône river to the Mediterraneen sea. J. Environ. radioact., 1990, 11, 105-139.

[9] PROGRAMME MIXTE FAO-OMS. - Codex Alimentarius (CAC, Vol. 17, suppl. 1), Genève : OMS, 1990. 